\title{
Emerging mupirocin resistance in methicillin-resistant Staphylococcus aureus isolates at a tertiary care children's hospital in Argentina
}

\author{
Nicolás M. Vázquez, B.S. ${ }^{a}$, Paulo Cáceres Guido, Pharmacist ${ }^{b}$, Graciela Fiorilli, Magister ${ }^{c}$ and Silvia Moreno, M.D. ${ }^{a}$
}

\begin{abstract}
In Latin America, few studies have been done in mupirocin resistance and biofilm formation in methicillin-resistant Staphylococcus aureus (MRSA). This study investigated mupirocin-resistance in MRSA isolates from pediatric patients with bacteremia and their ability to form biofilm. Antibiotic resistance was analyzed with the Kirby-Bauer test and the broth microdilution method. Bacterial biofilm formation was measured using the crystal violet assay. Among MRSA isolates, $2.3 \%(5 / 217)$ exhibited a high level of mupirocin-resistance with a minimum inhibitory concentration of $>512 \mu \mathrm{g} / \mathrm{mL}$, in addition to cross-resistance with clindamycin, erythromycin, gentamicin, and ciprofloxacin. Remarkably, biofilm formation in such isolates was moderate to high. This is the first report published in Argentina on clinical isolates of mupirocinresistant MRSA and it is critical for following its evolution over time at a local level and in the Latin American region. Key words: Staphylococcus aureus, resistance to drugs, mupirocin, biofilm, paediatrics.
\end{abstract}

http: / / dx.doi.org/10.5546/ aap.2019.eng.48

To cite: Vázquez NM, Cáceres Guido P, Fiorilli G, Moreno S. Emerging mupirocin resistance in methicillin-resistant Staphylococcus aureus isolates at a tertiary care children's hospital in Argentina. Arch Argent Pediatr 2019;117(1):48-52.

a. Laboratory of Active Ingredients from Plant Sources, Biomedical, Biotechnological, Environmental, and Diagnostic Study Center (Centro de Estudios Biomédicos, Biotecnológicos, Ambientales y de Diagnóstico, CEBBAD), CONICET, Pharmacy and Biochemistry degree. Universidad Maimónides.

b. Integrative Medicine Group. Hospital de Pediatría "J. P. Garrahan," Autonomous City of Buenos Aires, Argentina.

c. Department of Microbiology. Hospital de Pediatría "J. P. Garrahan," Autonomous City of Buenos Aires, Argentina.

E-mail address:Silvia Moreno,M.D.:smorenocontar@gmail.com

Funding: This research was funded by the National Scientific and Technical Research Council (Consejo Nacional de Investigaciones Científicas y Técnicas, CONICET). Grant:Multiannual Research Projects (PIP) 00894 (Silvia Moreno, M.D.) and Universidad Maimónides.

Conflict of interest: None.

Received: 3-23-2018

Accepted: 7-30-2018

\section{GLOSSARY}

CV: Crystal violet. COD: Critical OD.

MIC: Minimum inhibitory concentration.

MRSA: Methicillin-resistant Staphylococcus aureus.

MUP-R-MRSA: Mupirocin-resistant.

MUP-S-MRSA: Mupirocin-susceptible.

OD: Optical density.

\section{INTRODUCTION}

Common soft tissue staphylococcal infections are associated with high rates of morbidity and mortality in many countries worldwide, including Argentina. ${ }^{1,2}$ In particular, methicillin-resistant $S$. aureus (MRSA)-associated infections are lifethreatening, especially in immunocompromised hosts, because the bacteria spread from the skin to the lungs, the bloodstream or other body organs. For this reason, topical decolonization with an antimicrobial such as mupirocin is commonly done in hospitalized patients to prevent MRSA dissemination. ${ }^{3}$ However, the effectiveness of this treatment has reduced dramatically due to the emergence of resistant strains. ${ }^{4}$ Isolates with a low or high level of mupirocin resistance, which are defined as per the observed minimum inhibitory concentration (MIC) $(8-256 \mu \mathrm{g} / \mathrm{mL}$ or $>512 \mu \mathrm{g} /$ $\mathrm{mL}$, respectively), were observed. ${ }^{5}$ Another factor contributing to the success of $S$. aureus as a human pathogen in health care settings is its ability to form bacterial biofilm, multicellular communities adhered onto a surface, such as medical devices or tissues. Recurrent and chronic MRSA infections associated with the development of biofilm have a great impact on treatment schedules. ${ }^{6}$

Few studies have been done in Argentina and Latin America on the characterization of MRSA in pediatric patients. ${ }^{78}$ Likewise, much less is known on the presence of mupirocin resistance and its ability to form biofilm in MRSA. The objective of this study was to determine the presence of mupirocin-resistant MRSA (MUP-R-MRSA) and its ability to form biofilm among hospitalized patients in Hospital de Pediatría "J. P. Garrahan." 


\section{METHODOLOGY}

This was a descriptive study with blood sample analysis using a convenient sampling strategy of patients with bacteremia between January 2011 and December 2015. Hospital Garrahan is a leading children's hospital in Argentina and a regional referral center receiving children from neighboring countries like Bolivia, Peru, and Paraguay. It is located in the Autonomous City of Buenos Aires, Argentina. The study was approved by the Institutional Review Board and the Ethics Committee of Hospital Garrahan (protocol no. 868). One blood sample was collected per patient; $S$. aureus was identified at the laboratory of the Department of Microbiology of Hospital Garrahan according to the Clinical and Laboratory Standards Institute (CLSI) guidelines. ${ }^{9}$ MRSA was confirmed through the identification of specific protein profiles using a matrix-assisted laser desorption/ ionization time-of-flight (MALDI/TOF) mass spectrometry (bioMérieux, France). The antibiotic susceptibility of isolates was analyzed using the disc diffusion method for the following antibiotics: oxacillin, cefoxitin, erythromycin, clindamycin, teicoplanin, gentamicin, ciprofloxacin, levofloxacin, quinupristin/dalfopristina, linezolid, minocycline, rifampicin, and trimethoprimsulfamethoxazole, as per the recommendations and the classification of the European Committee on Antimicrobial Susceptibility Testing (EUCAST). Sensitivity tests were confirmed using VITEK 2 (bioMérieux). Mupirocin susceptibility (Forberton Co. Laboratories) was analyzed using the KirbyBauer disc diffusion method, while resistance was determined by measuring the MIC. The final concentrations were determined between 0.062 and $2.048 \mu \mathrm{g} / \mathrm{mL}$ using the broth microdilution method. ${ }^{10}$

Bacterial biofilm was detected using the crystal violet (CV) staining technique with culture in 96-well plates and determination of optical density (OD). Biofilm formation was estimated considering a critical OD (COD) of 0.1 , corresponding to the arithmetic mean of the absorbance of negative controls plus three times the standard deviation. ${ }^{11}$ Classification was as follows: non-biofilm-forming isolates $(\mathrm{OD} \leq \mathrm{COD})$; weak biofilm-forming isolates $(\mathrm{COD}<\mathrm{OD} \leq 2 \mathrm{COD})$; moderate biofilm-forming isolates ( $2 \mathrm{COD}<\mathrm{OD} \leq 4 \mathrm{COD})$; and strong biofilm-forming isolates (4 COD $<$ OD). Results were described as mean \pm standard deviation, and the statistical significance $(p<0.05)$ was determined using the InfoStat 2015 software.

\section{RESULTS}

A total of 217 clinical isolates of MRSA were analyzed; $2.3 \%(5 / 217)$ were resistant to mupirocin, as determined with the Kirby-Bauer technique (Table 1). In addition to oxacillinand cefoxitin-resistance, clinical isolates showed moderate resistance to erythromycin, clindamycin, and gentamicin $(27.6 \%, 25 \%$, and $15.2 \%$, respectively) and a low resistance to ciprofloxacin, levofloxacin, trimethoprimsulfamethoxazole, and linezolid $(6.9 \%, 1.4 \%$, $0.5 \%$, and $0.5 \%$, respectively).

Table 1 shows a summary of the cross-

TABLE 1. Antibiotic cross-resistance profiles and minimum inhibitory concentrations of mupirocin in methicillin-resistant Staphylococcus aureus clinical isolates

\begin{tabular}{|c|c|c|c|c|c|c|c|c|c|c|c|}
\hline \multirow[t]{3}{*}{ MRSA } & & & \multicolumn{9}{|c|}{ Antibiotic susceptibility } \\
\hline & \multicolumn{2}{|c|}{ Mupirocin } & Oxacillin & Cefoxitin & Erytromycin & Clindamycin & Gentamicin & Ciprofloxacin & Levofloxacin & Linezolid & Rifampicin \\
\hline & $\mathrm{KB}^{*}$ & $\mathrm{CIM}^{* *}$ & & & $\mathrm{~KB}^{*}$ & & & & & & \\
\hline GM-36 & $\mathrm{R}$ & $>1024$ & $\mathrm{R}$ & $\mathrm{R}$ & $\mathrm{R}$ & $\mathrm{R}$ & $S$ & $\mathrm{R}$ & $S$ & $S$ & S \\
\hline GM-42 & $\mathrm{R}$ & $\geq 512$ & $\mathrm{R}$ & $\mathrm{R}$ & $\mathrm{R}$ & $\mathrm{R}$ & $\mathrm{R}$ & $S$ & $S$ & $S$ & S \\
\hline GM-43 & $\mathrm{R}$ & $\geq 512$ & $\mathrm{R}$ & $\mathrm{R}$ & $\mathrm{R}$ & $\mathrm{R}$ & $\mathrm{R}$ & $S$ & $S$ & $S$ & $S$ \\
\hline GM-134 & $\mathrm{R}$ & $\geq 512$ & $\mathrm{R}$ & $\mathrm{R}$ & $\mathrm{R}$ & $\mathrm{R}$ & $\mathrm{R}$ & $S$ & $S$ & $S$ & $S$ \\
\hline GM-212 & $\mathrm{R}$ & $\geq 512$ & $\mathrm{R}$ & $\mathrm{R}$ & $\mathrm{R}$ & $\mathrm{R}$ & $\mathrm{R}$ & $\mathrm{S}$ & S & $\mathrm{S}$ & S \\
\hline GM-4 & S & 0.125 & $\mathrm{R}$ & $\mathrm{R}$ & $\mathrm{R}$ & $\mathrm{R}$ & $\mathrm{R}$ & $\mathrm{R}$ & I & S & $S$ \\
\hline GM-17 & $S$ & 0.125 & $\mathrm{R}$ & $\mathrm{R}$ & $\mathrm{R}$ & $\mathrm{R}$ & $\mathrm{R}$ & $\mathrm{R}$ & I & $S$ & I \\
\hline GM-20 & $S$ & 0.125 & $\mathrm{R}$ & $\mathrm{R}$ & $\mathrm{R}$ & $\mathrm{R}$ & $S$ & $S$ & $S$ & $S$ & $S$ \\
\hline GM-31 & S & 0.125 & $\mathrm{R}$ & $\mathrm{R}$ & $\mathrm{R}$ & $\mathrm{R}$ & $\mathrm{R}$ & $\mathrm{R}$ & I & $S$ & I \\
\hline GM-34 & $S$ & 0.250 & $\mathrm{R}$ & $\mathrm{R}$ & $\mathrm{R}$ & $\mathrm{R}$ & $\mathrm{R}$ & $\mathrm{R}$ & $\mathrm{R}$ & $\mathrm{R}$ & I \\
\hline
\end{tabular}

* Kirby-Bauer; ${ }^{* *}$ The MIC was measured using the microdilution technique ( $\left.\mu \mathrm{g} / \mathrm{mL}\right)$; MRSA: methicillin-resistant $S$. aureus; GM: sample from Hospital Garrahan; R: resistance; S: susceptibility; I: intermediate (resistance). 
resistance profiles of MUP-R-MRSA clinical isolates called MRSA GM-36, 42, 43, 134, and 212. It also includes mupirocin MIC values as determined with the broth microdilution method. All MUP-R-MRSA clinical isolates showed a MIC $>512 \mu \mathrm{g} / \mathrm{mL}$ and cross-resistance with clindamycin, erythromycin, gentamicin, and ciprofloxacin. In contrast, mupirocin-susceptible isolates measured under the same experimental conditions are shown and they exhibited expected MIC values for bacteria susceptible to mupirocin
$(0.12-0.25 \mu \mathrm{g} / \mathrm{mL})$.

In addition, MUP-R-MRSA isolates were tested for their biofilm formation ability. Results showed that all MUP-R-MRSA isolates (GM-36, 42, 43, 134, and 212) formed biofilm (Figure 1). Three of them formed a moderate amount of biofilm, whereas the other two formed strong biofilm. The analysis of the 5 mupirocin-susceptible isolates (MUP-SMRSA) that exhibited resistance to 4-9 antibiotics (GM-4, 17, 20, 31, and 34) showed that they were also able to form biofilm at a similar level than mupirocin-resistant isolates.

FIGURE 1. Detection of biofilm formation in mupirocin-resistant or susceptible methicillin-resistant Staphylococcus aureus clinical isolates with crystal violet staining at an absorbance of $595 \mathrm{~nm}$

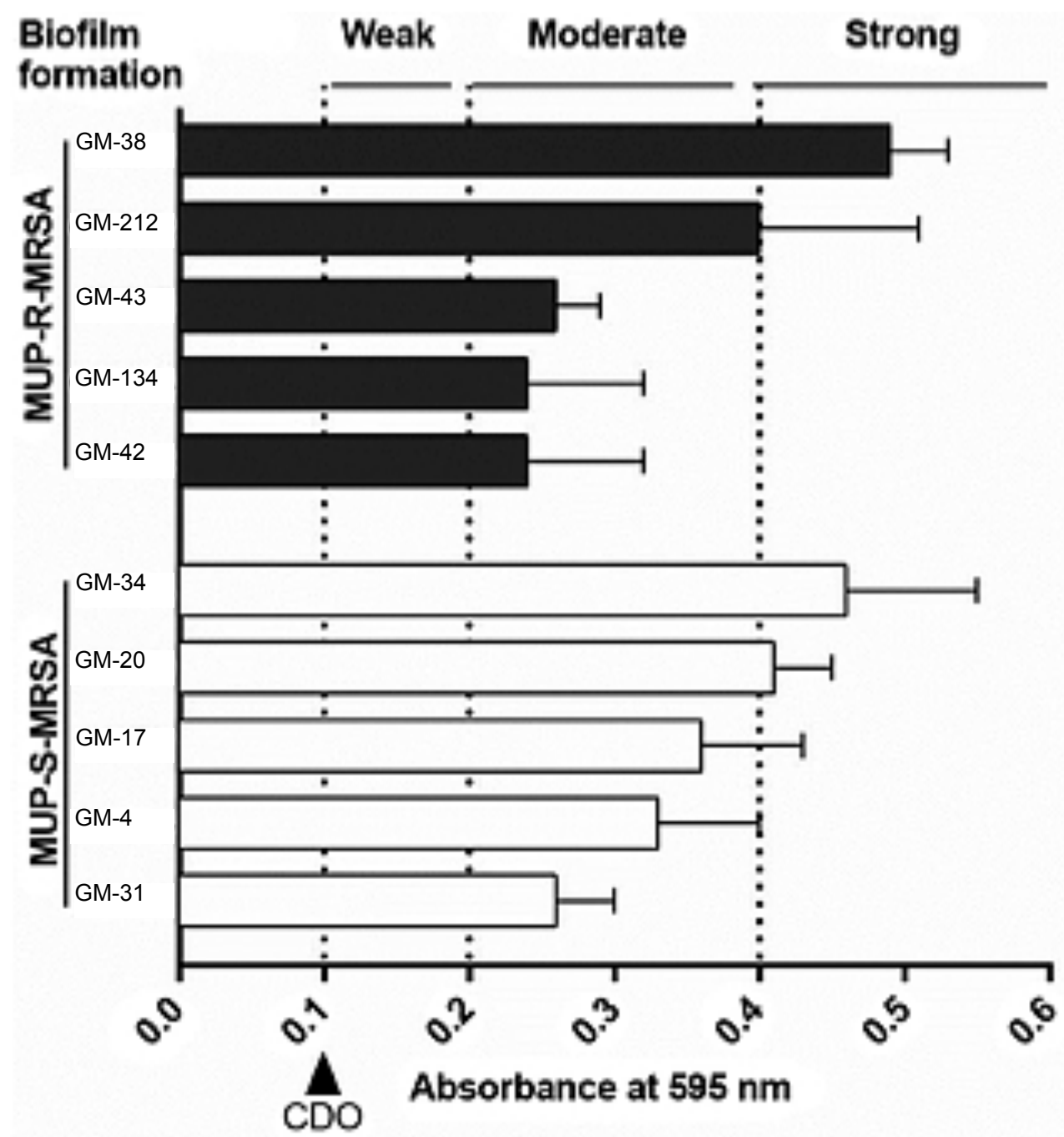

Biofilm formation was weak, moderate or strong based on a critical optical density $(C O D)=0.1$, shown as a vertical dotted line. Mupirocin-resistant or susceptible methicillin-resistant S. aureus (MRSA): MUP-R-MRSA or MUP-S-MRSA, respectively; COD: critical optical density; GM: Hospital Garrahan sample. 


\section{DISCUSSION}

This study reports, for the first time in Argentina, the emergence of mupirocin resistance in MRSA among pediatric patients. Although its incidence is low $(2.7 \%)$, it is worth noting the high level of mupirocin's cross-resistance with erythromycin, clindamycin, and gentamicin. To make things even more complicated, MUP-RMRSA isolates were able to synthesize biofilm and exhibited moderate-to-high levels of biofilm formation.

Other authors have reported a high level of simultaneous mupirocin resistance with other antibiotics in association with a more frequent MRSA colonization. ${ }^{4-12}$ Therefore, mupirocin is recommended as a first-line treatment for impetigo in patients with a limited number of lesions, especially considering MRSA local resistance patterns as per the 2014 guidelines of the Infectious Diseases Society of America. ${ }^{3}$ In a children's hospital in the USA, mupirocin resistance was detected in $14.7 \%$ of $S$. aureus isolates in association with recurrent skin and soft tissue infections, which was higher than the rate observed in other community studies conducted in children $(1.8 \%) .{ }^{4}$ In outpatients with skin diseases in the USA, mupirocin resistance was observed in $S$. aureus isolates in association with atopic dermatitis, epidermolysis bullosa, and immunosuppression as additional risk factors, with an incidence of 19.3-31.3\%. ${ }^{5}$

It is well known that, in patients with sepsis, medical devices such as central venous lines are commonly used and may favor bacterial biofilm formation. Our findings show that all MUP-RMRSA isolates formed biofilm. In relation to the possible correlation between mupirocin resistance in MRSA and its ability to form biofilm, other authors have reported biofilm-forming MUP-RMRSA strains isolated in swabs from infected wounds collected after a surgery. ${ }^{12}$ It has been suggested that biofilm formation may play a role in mupirocin resistance in the overall population of hospitalized patients. ${ }^{11,12}$

\section{CONCLUSION}

The presence of MUP-R-MRSA clinical isolates with ability to form biofilm in hospitalized patients is an obstacle for decolonization protocols and may result in chronic or persistent infections because they are capable of surviving in the host's hostile environment. Therefore, the emergence of mupirocin resistance in MRSA isolates in Argentina is an increasing problem that calls for the future monitoring of its evolution over time.

\section{Acknowledgments}

The authors would like to thank Claudia Hernández, M.D., Head of the Department of Microbiology of Hospital de Pediatría "J. P. Garrahan," for supplying MRSA clinical strains.

\section{REFERENCES}

1. Paganini HR, Della Latta $P$, Soto $A$, et al. Bacteriemias por Staphylococcus aureus adquiridas en la comunidad: 17 años de experiencia en niños de la Argentina. Arch Argent Pediatr. 2010; 108(4):311-7.

2. Khokhlova OE, Hung WC, Wan TW, et al. Healthcare- and community-associated methicillin-resistant Staphylococcus aureus (MRSA) and fatal pneumonia with pediatric deaths in Krasnoyarsk, Siberian Russia: Unique MRSA's multiple virulence factors, genome, and stepwise evolution. PLoS One. 2015; 10(6):e0128017.

3. Stevens DL, Bisno AL, Chambers HF, et al. Practice guidelines for the diagnosis and management of skin and soft tissue infections: 2014 Update by the Infectious diseases society of America. Clin Infect Dis. 2014; 59(2):147-59.

4. Hogue JS, Buttke P, Braun LE, Fairchok MP. Mupirocin resistance related to increasing mupirocin use in clinical isolates of methicillin-resistant Staphylococcus aureus in a pediatric population. J Clin Microbiol. 2010; 48(7):2599-600.

5. Antonov NK, Garzon MC, Morel KD, et al. High prevalence of mupirocin resistance in Staphylococcus aureus isolates from a pediatric population. Antimicrob Agents Chemother. 2015; 59(6):3350-6.

6. McCarthy H, Rudkin JK, Black NS, et al. Methicillin resistance and the biofilm phenotype in Staphylococcus aureus. Front Cell Infect Microbiol. 2015; 5:1.

7. Gardella N, Murzicato S, Di Gregorio S, et al. Prevalence and characterization of methicillin-resistant Staphylococcus aureus among healthy children in a city of Argentina. Infect Genet Evol. 2011; 11(5):1066-71.

8. Ramos RL, Teixeira LA, Ormonde LR, et al. Emergence of mupirocin resistance in multiresistant Staphylococcus aureus clinical isolates belonging to Brazilian epidemic clone III: B:A. J Med Microbiol. 1999; 48(3):303-7.

9. Clinical and Laboratory Standards Institute. Performance for antimicrobial susceptibility testing. 26th ed. Wayne, PA: CLSI; 2016.

10. Ojeda-Sana AM, Repetto V, Moreno S. Carnosic acid is an efflux pumps modulator by dissipation of the membrane potential in Enterococcus faecalis and Staphylococcus aureus. World J Microbiol Biotechnol. 2013; 29(1):137-44.

11. Xu Z, Liang $Y$, Lin S, et al. Crystal Violet and XTT Assays on Staphylococcus aureus Biofilm Quantification. Curr Microbiol 2016; 73(4):474-82.

12. Günther F, Blessing B, Tacconelli E, Mutters N. MRSA decolonization failure-are biofilms the missing link? Antimicrob Resist Infect Control. 2017; 6:32. 